\title{
QCD Corrections to Supersymmetric Higgs Boson Production: The Role of Squark Loops
}

\author{
S. Dawson, ${ }^{1}$ A. Djouadi, ${ }^{2}$ and M. Spira ${ }^{3}$ \\ ${ }^{1}$ Department of Physics, Brookhaven National Laboratory, Upton, New York 11973-5000 \\ ${ }^{2}$ Institut für Theoretische Physik, Universität Karlsruhe, D-76128 Karlsruhe, Germany \\ ${ }^{3}$ CERN, TH Division, CH-1211 Geneva 23, Switzerland
}

(Received 27 March 1996)

\begin{abstract}
We calculate the two-loop QCD corrections to the production of the neutral supersymmetric Higgs bosons via the gluon fusion mechanism at hadron colliders, including the contributions of squark loops. To a good approximation, these additional contributions lead to the same QCD corrections as in the case where only $t$ and $b$ quark loops are taken into account. The inclusion of both squark loops and QCD corrections increase the Higgs production cross sections significantly over the lowest order rates. [S0031-9007(96)00512-1]
\end{abstract}

PACS numbers: 14.80.Cp, 12.38.Bx, 12.60.Jv, 13.85.Qk

The search for Higgs particles is an important component of the experimental program at future high energy hadron colliders. As such, it is vital to have reliable predictions for the production rates both in the standard model (SM) and in the minimal supersymmetric extension of the standard model (MSSM). The two-loop QCD corrections to the main production process, the gluon fusion mechanism [1], have been calculated in the SM in Refs. [2,3] and later generalized to the quark contributions in the MSSM [3,4]. The corrections are large and positive, increasing the production rates significantly.

The MSSM requires the introduction of two Higgs doublets leading, after spontaneous symmetry breaking, to two neutral $C P$-even ( $h$ and $H$ ), a neutral $C P$-odd $(A)$, and two charged $\left(H^{ \pm}\right)$Higgs particles [5]. While in the SM the dominant contribution to Higgs boson production in the gluon fusion mechanism originates from top and, to a lesser extent, bottom quark loops, in the MSSM there are additional contributions to the production of the $C P$-even Higgs bosons from scalar squark loops. These contributions can be neglected for very heavy squarks. However, many supergravity-inspired models predict squark (in particular, stop and sbottom squark) masses significantly below $1 \mathrm{TeV}$ [6]. In this case, squark loop contributions to the Higgs-gluon couplings can be of the same order, or even larger, as the standard quark contributions, as was recently stressed in Ref. [7].

In this Letter, we present the $\mathcal{O}\left(\alpha_{s}^{3}\right)$ QCD corrections to the cross sections $\sigma(p p \rightarrow \mathcal{H}+X)$ of the fusion processes for the neutral $C P$-even Higgs particles $\mathcal{H}=$ $h, H$

$$
g g \longrightarrow \mathcal{H}(g) \text { and } \quad g q \longrightarrow \mathcal{H} q, \quad q \bar{q} \longrightarrow \mathcal{H} g
$$

Because of CP invariance, squark loops do not contribute to the production of the $C P$-odd Higgs boson in lowest order. The QCD corrections from squark loops are evaluated in the heavy squark limit, where the calculation can be simplified by extending the lowest-order low-energy theorems $[3,8]$ to two loops. This limit should be a very good ap- proximation [3] for the production of Higgs particles with masses smaller than twice the squark masses. Given the experimental bounds on the squark masses [9], this is fully justified in the case of the lightest Higgs boson $h$, which is constrained to be lighter than $\sim 130 \mathrm{GeV}$ in the MSSM; for the heavier $C P$-even Higgs boson $H$, this approximation is valid for masses smaller than a few hundred GeV. For simplicity, we will restrict ourselves to the case of degenerate squarks where mixing effects are absent (in the absence of gluino exchange, the results can be trivially generalized to include mixing). Also in this case, scalar squarks will not contribute to the production of the $C P$-odd Higgs boson $A$ at next-to-leading order.

To lowest order, the cross sections for $C P$-even Higgs boson production at proton colliders are given by

$$
\sigma_{L O}(p p \longrightarrow \mathcal{H}+X)=\sigma_{0}^{\mathcal{H}} \tau_{\mathcal{H}} \frac{d \mathcal{L} g g}{d \tau_{\mathcal{H}}},
$$

with $d \mathcal{L}^{g g} / d \tau_{\mathcal{H}}$ the gluon luminosity at $\tau_{\mathcal{H}}=M_{\mathcal{H}}^{2} / s$ and $s$ is the total c.m. energy. The parton cross sections are built up from heavy quark and squark amplitudes,

$$
\sigma_{0}^{\mathcal{H}}=\frac{G_{F} \alpha_{s}^{2}}{128 \sqrt{2} \pi}\left|\sum_{Q} g_{Q}^{\mathcal{H}} A_{Q}^{\mathcal{H}}\left(\tau_{Q}\right)+\sum_{\tilde{Q}} g_{\tilde{Q}}^{\mathcal{H}} A_{\tilde{Q}}^{\mathcal{H}}\left(\tau_{\tilde{Q}}\right)\right|,
$$

where the sums run over $t, b$ quarks and the left- and right-handed squarks $\tilde{Q}_{L}, \tilde{Q}_{R}$, which, in the absence of mixing, are identical to the mass eigenstates. The form factors, with the scaling variables $\tau_{Q / \tilde{Q}} \equiv 4 m_{Q / \tilde{Q}}^{2} / M_{\mathcal{H}}^{2}$, can be expressed as

$$
\begin{gathered}
A_{Q}\left(\tau_{Q}\right)=\tau_{Q}\left[1+\left(1-\tau_{Q}\right) f\left(\tau_{Q}\right)\right], \\
A_{\tilde{Q}}\left(\tau_{\tilde{Q}}\right)=-\frac{1}{2} \tau_{\tilde{Q}}\left[1-\tau_{\tilde{Q}} f\left(\tau_{\tilde{Q}}\right)\right],
\end{gathered}
$$

using the scalar triangle integral

$$
f(\tau)= \begin{cases}\arcsin ^{2}\left(\frac{1}{\sqrt{\tau}}\right), & \tau \geq 1, \\ -\frac{1}{4}\left[\ln \frac{1+\sqrt{1-\tau}}{1-\sqrt{1-\tau}}-i \pi\right]^{2}, & \tau<1 .\end{cases}
$$


The normalized scalar quark and squark couplings to the $C P$-even Higgs bosons, $g_{Q, \tilde{Q}}^{\mathcal{H}}$, can be found in Refs. [3,5]. In the case where all squarks are taken to be degenerate, only the contributions proportional to the Yukawa-type couplings of the stop and sbottom squarks have to be added to the top and bottom quark loop contributions. The couplings, as well as the $C P$ even Higgs masses, are determined at tree level by two parameters, which are generally chosen to be the ratio of the vacuum expectation values of the two Higgs fields, $\tan \beta$, and the pseudoscalar Higgs mass, $M_{A}$. All MSSM Higgs masses and couplings are calculated using the two-loop renormalization group improved effective potential [10].

The QCD corrections to the gluon fusion process, Eq. (1), consist of virtual two-loop corrections and oneloop real corrections due to gluon radiation, as well as contributions from quark-gluon initial states and quarkantiquark annihilation. The renormalization program has been carried out in the minimal subtraction ( $\overline{\mathrm{MS}}$ ) scheme for the strong coupling constant and the parton densities, while the quark and squark masses are defined at the poles of their respective propagators. The result for the cross sections can be cast into the form

$$
\begin{aligned}
\sigma(p p \longrightarrow \mathcal{H}+X)= & \sigma_{0}^{\mathcal{H}}\left[1+C_{\mathcal{H}}\left(\tau_{Q}, \tau_{\tilde{Q}}\right) \frac{\alpha_{s}}{\pi}\right] \\
& \times \tau_{\mathcal{H}} \frac{d \mathcal{L} g g}{d \tau_{\mathcal{H}}}+\Delta \sigma_{g g}^{\mathcal{H}} \\
& +\Delta \sigma_{g g}^{\mathcal{H}}+\Delta \sigma_{q \bar{q}}^{\mathcal{H}} .
\end{aligned}
$$

The coefficient $C_{\mathcal{H}}$ denotes the virtual two-loop corrections regularized by the infrared singularities of the real gluon emission. The terms $\Delta \sigma_{i j}^{\mathcal{H}}(i, j=g, q)$ denote the finite parts of the real corrections due to gluon radiation and the $g q$ and $q \bar{q}$ initial states. The expressions for the $t, b$ quark contribution can be found in Refs. [2-4].

The calculation of the QCD corrections has been performed by extending the low-energy theorems $[3,8]$ to scalar squarks at the two-loop level. For a light $C P$-even Higgs boson, these theorems relate the matrix elements of the quark and squark contributions to the Higgs-gluon vertex to the gluon two-point functions. Denoting the matrix element of the squark contribution to the gluon two-point function by $\mathcal{M}_{\tilde{Q}}(g g)$ and the corresponding matrix elements with an additional light $C P$-even Higgs boson by $\mathcal{M}_{\tilde{Q}}(\operatorname{gg} \mathcal{H})$, one has at lowest order [11]

$$
\begin{aligned}
\mathcal{M}_{\tilde{Q}}(g g \mathcal{H})= & \sum_{\tilde{Q}}\left(\sqrt{2} G_{F}\right)^{1 / 2} \\
& \times g_{\tilde{Q}}^{\mathcal{H}} m_{\tilde{Q}} \frac{\partial \mathcal{M}_{\tilde{Q}}(g g)}{\partial m_{\tilde{Q}}} .
\end{aligned}
$$

To extend this relation to higher orders, all quantities have to be replaced by their bare values; after differentiation, the renormalization then has to be performed. In the following, we consider only the pure gluon exchange contributions, which are expected to be the dominant ones; for heavy enough gluinos, the two-loop corrections due to gluino exchange should be small since they are suppressed by inverse powers of the gluino mass. In this case, the differentiation with respect to the bare squark mass $m_{\tilde{Q}}^{0}$ can be rewritten in terms of the renormalized mass $m_{\tilde{Q}}$. A finite contribution to the QCD corrections arises from the anomalous mass dimension $\gamma_{\tilde{Q}}[3]$,

$$
m_{\tilde{Q}}^{0} \frac{\partial}{\partial m_{\tilde{Q}}^{0}}=\frac{m_{\tilde{Q}}}{1+\gamma_{\tilde{Q}}} \frac{\partial}{\partial m_{\tilde{Q}}} .
$$

The remaining differentiation with respect to the renormalized squark mass of the gluon two-point function leads to the squark contribution $\beta_{\tilde{Q}}$ to the QCD $\beta$ function. The final result for the squark contributions to the $\mathcal{H}$ coupling to gluons can be expressed in terms of the effective Lagrangian

$$
\begin{aligned}
\mathcal{L}_{\mathrm{eff}}^{\tilde{Q}}= & \left(\sqrt{2} G_{F}\right)^{1 / 2} \sum_{\tilde{Q}} \frac{g_{\tilde{Q}}^{\mathcal{H}}}{4} \frac{\beta_{\tilde{Q}}\left(\alpha_{s}\right) / \alpha_{s}}{1+\gamma_{\tilde{Q}}\left(\alpha_{s}\right)} \\
& \times G^{a \mu \nu} G_{\mu \nu}^{a} \mathcal{H} .
\end{aligned}
$$

The QCD corrections are then fully determined by the anomalous mass dimension of the squarks $[6,12]$

$$
\gamma_{\tilde{Q}}=\frac{4}{3} \frac{\alpha_{s}}{\pi}+\mathcal{O}\left(\alpha_{s}^{2}\right)
$$

and the squark contribution to the QCD $\beta$ function [13]

$$
\frac{\beta_{\tilde{Q}}\left(\alpha_{s}\right)}{\alpha_{s}}=\frac{\alpha_{s}}{12 \pi}\left[1+\frac{11}{2} \frac{\alpha_{s}}{\pi}\right]+\mathcal{O}\left(\alpha_{s}^{3}\right),
$$

resulting in a final rescaling of the lowest-order Lagrangian by a factor $1+25 \alpha_{s} / 6 \pi$ at next-to-leading order, compared to a rescaling factor $1+11 \alpha_{s} / 4 \pi$ for the quark contribution. Starting from the Lagrangian Eq. (10), the effective QCD corrections due to real gluon emission and the $g q / q \bar{q}$ initial states have to be added. These corrections are identical to the corresponding corrections to quark loops [3] in the heavy quark limit.

The QCD corrected squark loop amplitudes have to be added coherently to the corrected $t, b$ loop amplitudes, whose full mass dependence is known. To obtain a more reliable prediction for the total cross sections, the resulting amplitudes for the squark contributions have been normalized to the lowest-order amplitude in the limit of large squark masses. These ratios are then multiplied by the lowest-order amplitude, including the full squark mass dependence. The heavy squark limit is then expected to be a very good approximation for Higgs masses below the $\tilde{Q} \tilde{Q}^{*}$ threshold, as in the corresponding case of top quark contributions [3].

The final results for the partonic cross sections defined in Eq. (7) can be expressed as 


$$
\begin{aligned}
C_{\mathcal{H}}\left(\tau_{Q}, \tau_{\tilde{Q}}\right) & =\pi^{2}+c_{1}^{\mathcal{H}}\left(\tau_{Q}, \tau_{\tilde{Q}}\right)+\frac{33-2 N_{F}}{6} \ln \frac{\mu^{2}}{M_{\mathcal{H}}^{2}} \\
\Delta \sigma_{g g}^{\mathcal{H}} & =\int_{\tau_{\mathcal{H}}}^{1} d \tau \frac{d \mathcal{L} g g}{d \tau} \frac{\alpha_{s}}{\pi} \sigma_{0}^{\mathcal{H}}\left\{-\hat{\tau} P_{g g}(\hat{\tau}) \ln \frac{M^{2}}{\tau_{s}}+d_{g g}^{\mathcal{H}}\left(\hat{\tau}, \tau_{Q}, \tau_{\tilde{Q}}\right)+6\left[1+\hat{\tau}^{4}+(1-\hat{\tau})^{4}\right]\left(\frac{\ln (1-\hat{\tau})}{1-\hat{\tau}}\right)_{+}\right\} \\
\Delta \sigma_{g q}^{\mathcal{H}} & =\int_{\tau_{\mathcal{H}}}^{1} d \tau \sum_{q, \bar{q}} \frac{d \mathcal{L} g q}{d \tau} \frac{\alpha_{s}}{\pi} \sigma_{0}^{\mathcal{H}}\left\{d_{g q}^{\mathcal{H}}\left(\hat{\tau}, \tau_{Q}, \tau_{\tilde{Q}}\right)-\frac{\hat{\tau}}{2} P_{g q}(\hat{\tau})\left[\ln \frac{M^{2}}{\tau s}-2 \ln (1-\hat{\tau})\right]\right\} \\
\Delta \sigma_{q \bar{q}}^{\mathcal{H}} & =\int_{\tau_{\mathcal{H}}}^{1} d \tau \sum_{q} \frac{d \mathcal{L}{ }^{q \bar{q}}}{d \tau} \frac{\alpha_{s}}{\pi} \sigma_{0}^{\mathcal{H}} d_{q \bar{q}}^{\mathcal{H}}\left(\hat{\tau}, \tau_{Q}, \tau_{\tilde{Q}}\right)
\end{aligned}
$$

with $\hat{\tau}=\tau_{\mathcal{H}} / \tau$ and $N_{F}$ being the number of light flavors contributing to the evolution of $\alpha_{s}$ and the parton densities. The renormalization scale $\mu$ enters the lowestorder expression $\sigma_{0}^{\mathcal{H}}$ as the scale of the strong coupling $\alpha_{s}=\alpha_{s}(\mu)^{2} . \quad P_{g g}, P_{g q}$ denote the Altarelli-Parisi splitting functions [14]; $M$ is the factorization scale at which the parton luminosities are evaluated. $F_{+}$is the usual + distribution, $F(\hat{\tau})_{+}=F(\hat{\tau})-\delta(1-\hat{\tau}) \int_{0}^{1} d x F(x)$.

The contributions to the coefficients $c_{1}^{\mathcal{H}}$ and $d_{i j}^{\mathcal{H}}$ appearing in Eq. (13) from squarks, in the heavy squark limit without $t, b$ loops, are given by

$$
\begin{gathered}
c_{1}^{\mathcal{H}} \longrightarrow \frac{25}{3}, \quad d_{g g}^{\mathcal{H}} \longrightarrow-\frac{11}{2}(1-\hat{\tau})^{3}, \\
d_{g q}^{\mathcal{H}} \longrightarrow-1+2 \hat{\tau}-\frac{1}{3} \hat{\tau}^{2}, \quad d_{q \bar{q}}^{\mathcal{H}} \longrightarrow \frac{32}{27}(1-\hat{\tau})^{3} .
\end{gathered}
$$

In Fig. 1, we present the $K$ factors for the QCD corrections to the production of the $C P$-even MSSM Higgs bosons as functions of the $\mathcal{H}$ masses for the LHC at a c.m. energy $\sqrt{s}=14 \mathrm{TeV}$ with (solid lines) and without (dashed lines) the squark contributions. The $K$ factors are defined as the ratios of the QCD corrected and lowest-order cross sections, using next-to-leading order $\alpha_{s}$ and parton densities in both terms. A common value, $m_{\tilde{Q}}=200 \mathrm{GeV}$, has been used for the left- and righthanded stop and sbottom squark masses [15]. This value is identified with the SUSY scale of the MSSM couplings and Higgs masses. The QCD corrections to the squark loops are large, approximately of the same size as the QCD corrections to the quark loops.

The QCD corrections enhance the cross sections by a factor between 1.6 and 2.8, if the lowest-order cross sections are evolved with next-to-leading order $\alpha_{s}$ and parton densities. If the lowest-order cross sections are convoluted with lowest order $\alpha_{s}$ and parton densities, the $K$ factors are reduced to a level between 1 and 2 . It can be inferred from Fig. 1 that the inclusion of squark loops in the production of both $C P$-even Higgs particles $h$ and $H$ does not substantially modify the $K$ factors compared to the case where squark loops are absent. The $\mathcal{O}(10 \%)$ discrepancy between the two factors for small $\beta$ (where both the top and the stop contributions are dominant and can be approximated by their heavy mass limits) is due mainly to the difference between the contribution of quarks and squarks to the effective Lagrangian $\left(c_{1}^{\mathcal{H}}=\frac{25}{3}\right.$ for $\tilde{Q}$ and $c_{1}^{\mathcal{H}}=\frac{11}{2}$ for $Q$ loops).

We have verified that the $K$ factors do not depend significantly on the squark mass which enters the MSSM couplings and lowest-order cross sections in our analysis. In fact, in the extreme situation where one of the $\tilde{t}, \tilde{b}$ squark eigenstates is relatively light while the other squarks are heavy and decouple (as is the case for large squark mixing), the $K$ factors are almost the same as in Fig. 1. Therefore, while it substantially changes the Higgs-squark couplings and hence the production cross sections, mixing in the stop or sbottom sectors should have a rather modest impact on the $K$ factors.

Thus, to a good approximation, the effect of the squark loops in the gluon fusion mechanism is quantitatively determined by the lowest-order cross section (including squark loop contributions), multiplied by the known $K$ factors when only the $t, b$ quark contributions $[3,4]$ are taken into account.

In Fig. 2, we illustrate the effect of including the squark loops and the QCD corrections to the production rate of the lightest $C P$-even Higgs particle. The ratio of the QCD

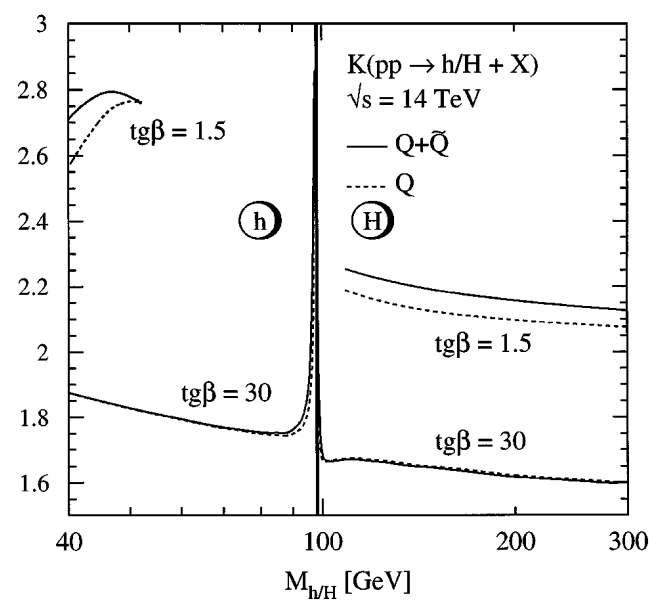

FIG. 1. Ratio of the QCD corrected cross section to the lowest-order result for $\sigma(p p \rightarrow \mathcal{H}+X)$ for $\tan \beta=1.5$ and 30. Solid lines include $t, b$ and squark contributions (with $m_{\tilde{Q}}=200 \mathrm{GeV}$ ); dashed lines include only the $t, b$ contributions. $m_{b}=5 \mathrm{GeV}, m_{t}=176 \mathrm{GeV}$, and $\alpha_{s}\left(M_{Z}^{2}\right)=$ 0.118. We use next-to-leading order GRV parton densities [16] and take the renormalization scale $\mu$ and the factorization scale $M$ equal to $M_{h / H}$. 


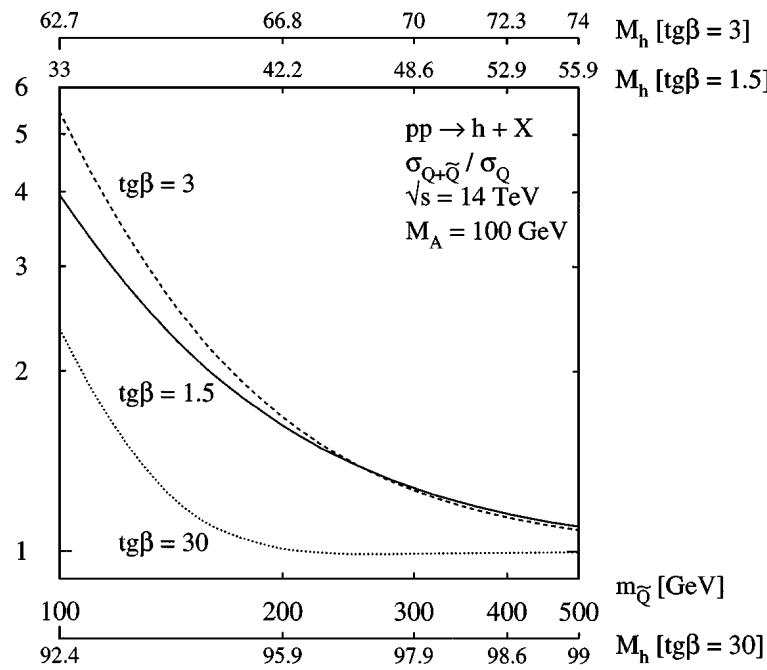

FIG. 2. Ratio of the QCD corrected cross sections $\sigma(p p \rightarrow$ $h+X)$ with and without squark loops for three values of $\tan \beta=1.5,3,30$, and for $M_{A}=100 \mathrm{GeV}$. The secondary axes present the corresponding Higgs masses $M_{h}$. The quark masses $\alpha_{s}$ and the parton densities are as in Fig. 1 .

corrected cross sections with and without squark loops is shown as a function of the common squark mass for three values of $\tan \beta=1.5,3$, and 30 , with the pseudoscalar Higgs mass fixed to $M_{A}=100 \mathrm{GeV}$. The squark contributions increase the production cross sections significantly for squark masses below about $500 \mathrm{GeV}$, especially for small and moderate values of $\tan \beta$; for higher masses $m_{\tilde{Q}}$, the squarks decouple from the amplitude. This large effect can be understood by recalling that, for these values of $\tan \beta$, the top and stop contributions dominate, and the inclusion of the stop loops leads, in the heavy top and squark limit, to the enhancement of the lowest-order production cross section by an amount

$$
\frac{\sigma_{t+\tilde{t}}}{\sigma_{t}}=\left(1+\frac{1}{2} \frac{m_{t}^{2}}{m_{\tilde{t}}^{2}}\right)^{2},
$$

where $\sigma_{t+\tilde{t}}\left(\sigma_{t}\right)$ denotes the cross section including (without) stop loops. Thus, for stop masses $m_{\tilde{t}}$ of the order of the top mass $m_{t}$, the cross sections are significantly enhanced by including stop loops. For large values of $\tan \beta$, because the Higgs couplings to $(s)$ top $(s)$ quarks are strongly suppressed (except for $h$ in the decoupling limit), and the contribution of the bottom loop is enhanced by large logarithms compared to the sbottom loop, the production cross section is significantly affected only by light squark contributions, while they become negligible for sbottom masses of the order of $200 \mathrm{GeV}$.

Light squarks, with masses, $m_{\tilde{Q}} \lesssim 300 \mathrm{GeV}$, can have a large impact on the production cross sections of the $C P$-even MSSM Higgs bosons at hadron colliders. We have presented the QCD corrections to the gluon fusion processes $p p \rightarrow g g \rightarrow h / H$ including squark loops, in the heavy squark limit, which should well describe the full corrections including mass effects, at least in the range where the Higgs masses are smaller than twice the squark masses. The $t$ and $b$ quark mass dependence has been included exactly. To a good approximation, the $K$ factors are the same as the corresponding $K$ factors when only top and bottom contributions are included. Since they increase the production cross sections significantly, the QCD corrections must be taken into account along with squark loops for $m_{\tilde{Q}} \lesssim 300 \mathrm{GeV}$.

Discussions with Jon Bagger and Manuel Drees about SUSY beta functions and Jim Wells about Ref. [7] are gratefully acknowledged. S. D. is supported by the U.S. Department of Energy under Contract DE-AC0276CH00016. A. D. is supported by the DFG, Bonn.

[1] H. M. Georgi, S. I. Glashow, M.E. Machacek, and D. V. Nanopoulos, Phys. Rev. Lett. 40, 692 (1978).

[2] S. Dawson, Nucl. Phys. B359, 283 (1991); A. Djouadi, M. Spira, and P. M. Zerwas, Phys. Lett. B 264, 440 (1991); D. Graudenz, M. Spira, and P. M. Zerwas, Phys. Rev. Lett. 70, 1372 (1993); M. Spira, Report No. DESY T-95-05, hep-ph/9510347 (to be published).

[3] M. Spira, A. Djouadi, D. Graudenz, and P.M. Zerwas, Nucl. Phys. B453, 17 (1995).

[4] M. Spira, A. Djouadi, D. Graudenz, and P. M. Zerwas, Phys. Lett. B 318, 347 (1993).

[5] For a review on the MSSM see, e.g., J. Gunion, H. E. Haber, G. Kane, and S. Dawson, The Higgs Hunter's Guide (Addison-Wesley, Redwood City, 1990); J. Bagger, TASI-91 (World Scientific, Singapore, 1992).

[6] See, for instance, V. Barger, M. Berger, and P. Ohmann, Phys. Rev. D 47, 1093 (1994).

[7] G. Kane, G. Kribs, S. Martin, and J. Wells, Phys. Rev. D 53, 213 (1996); B. Kileng, P. Osland, and P. Pandita, Report No. hep-ph/9601284 (to be published).

[8] J. Ellis, M. K. Gaillard, and D. V. Nanopoulos, Nucl. Phys. B106, 292 (1976); A. Vainshtein, M. Voloshin, V. Zakharov, and M. Shifman, Sov. J. Nucl. Phys. 30, 711 (1979); B. Kniehl and M. Spira, Z. Phys. C 69, 77 (1995).

[9] CDF Collaboration, F. Abe et al., Phys. Rev. Lett. 75, 613 (1995); D0 Collaboration, S. Abachi et al., Phys. Rev. Lett. 75, 618 (1995); J. J. Hernandez, Proceedings of the La Thuile Conference, 1996, edited by G. Bellettini and M. Greco (to be published).

[10] M. Carena, J. Espinosa, M. Quiros, and C. Wagner, Phys. Lett. B 355, 209 (1995).

[11] Note that Eq. (8) is only valid if four-point interactions between Higgs bosons and squarks do not contribute.

[12] L. Alvarez-Gaumé, J. Polchinski, and M. Wise, Nucl. Phys. B221, 495 (1983); S. Martin and M. Vaughn, Phys. Rev. D 50, 2282 (1994); J. Derendinger, and C. Savoy, Nucl. Phys. B237, 307 (1984).

[13] W. Caswell, Phys. Rev. Lett. 33, 244 (1974); D. R. T. Jones Phys. Rev. D 25, 581 (1982); M. Einhorn and D. R. T. Jones, Nucl. Phys. B196, 475 (1982).

[14] G. Altarelli and G. Parisi, Nucl. Phys. B126, 298 (1977).

[15] Current limits on SUSY particle masses can be found in J.-F. Grivaz, Proceedings of the 1995 EPS Conference, Brussels, 1995 (unpublished).

[16] M. Glück, E. Reya, and A. Vogt, Z. Phys. C 53, 127 (1992). 